\title{
Speeding up N-body Calculations on Machines without Hardware Square Root
}

\author{
ALAN H. KARP \\ IBM Scientific Center, Palo Alto, CA 94304
}

\begin{abstract}
The most time consuming part of an $\mathrm{N}$-body simulation is computing the components of the accelerations of the particles. On most machines the slowest part of computing the acceleration is in evaluating $r^{-3 / 2}$, which is especially true on machines that do the square root in soffware. This note shows how to cut the time for this part of the calculation by a factor of 3 or more using standard Fortran. c) 1993 John Wiley \& Sons, Inc.
\end{abstract}

\section{INTRODUCTION}

Many phenomena in astmphysics and chemistry are being simulated using $\mathrm{N}$-body methods [1.2. The most time consuming part of such simulations is computing the accelerations on each particle due to all the others. This is true for the simple 1"2 merhods. tree-based methods [3]. or those using neighbor lists $[\mathbf{t}]$.

If the potential heing used has an odd porrer of the particle separation in it. computing the orthogonal components of the acceleration will inwolve taking a square root. Although some machines do square root in hardware. many do not. It is not unusual to find that half the run time of an N-body calculation is spent in the square root subroutine.

In our case. we nant to evaluate the acceleration on each particle in a system of self-gravitating bodies. For example. the $x$-component of the ac-

\footnotetext{
Received (octotice 1902)

Accepted November 1992

Alan Karp's present address: llentett-Packard Labs 3[ -?. 1501 Patre Mill Road. Pato . lto. (A 9+30+. karp@ohpl.hp.com

(C) 199:3 by John Wiley d Sons. Inc.

Scientific Programming. Vol. 1. pp. 13:3-1+0 (1992

(c.ci $1058-9)+4 / 93 / 0201333-08$
}

celeration for particle $j$ under the gravitational influence of particle $k$ is

$$
\hat{\mathbf{i}} \cdot \mathbf{a}_{j k}=\frac{C m_{k}\left(x_{j}-x_{k}\right)}{r^{3}}
$$

where $\hat{\mathbf{i}}$ is the unit vector in the $\boldsymbol{x}$ direction. $G$ is the gravitational constant. $m_{k}$ is the mass of particle $k$. and $r$ is the separation between the particles.

$$
r=\sqrt{\left(x_{j}-x_{k}\right)^{2}+\left(y_{j}-y_{k}\right)^{2}+\left(z_{j}-z_{k}\right)^{2}}
$$

For efficiency. we usually code $r^{3}$ as $r^{2} \sqrt{r^{2}}$.

The system square root routine. not knowingr how its result will be used. computes the square root with a divide-free Newton iteration to compute the inverse of the square root followed by a muliplication by the input value to get the final result. The compiler then multiplies by $r^{2}$ and divides the result into the numerator. Because both divisions and square roots are usually slow, this operation rakes a long time.

Table 1 show's the time needed for some common operations-an empty loop. a simple assignment division. square root and $x^{-3 / 2}$. All times are in machine cycles per element measured on an IBII RISC System/6000 Model 500. Because RISC machines of this kind usually improve per- 
Table 1. Time for Some Common Operations in Machine Cycles Per Element Run on an IB.I RS/6000-540

\begin{tabular}{lcc}
\hline$m$ & Rolled & Lnrolled $^{*}$ \\
\hline Empty & 2 & 2 \\
assignment & 4 & 4 \\
$x^{-1}$ & 20 & 20 \\
$\sqrt{x}$ & 56 & 56 \\
$(x \sqrt{x})^{-1}$ & 77 & 77 \\
\hline
\end{tabular}

* Unrolled refers to loops unrolled eight ways. Note that loop unrolling has no effect.

formance by pipelining arithmetic operations. unrolling loops frequently speeds things up. Clearly. the operations measured do not benefit.

It is possible to do considerably better than the direct computation. This note shows how to use standard Fortran to evaluate the acceleration in about one third the time taken by the direct evaluation.

\section{ALGORITHM}

The only way we can beat the efficiency of the system routines is to use our extra knowledge of the problem. In this case, we nill not compute $\sqrt{r^{2}}$. Instead, we will compute $r^{-3}$ directly from $r^{2}$.

The simplest approach is to approximate this function with a polynomial. Chebychev polynomials are frequently used because they minimize the maximum error of the approximation on some interval $[5]$. One difficulty is that the approximation is accurate only if the arguments are limited to a relatively small range. With a range reduction the procedure for an input argument $r^{2}$ becomes

1. Find a $u$ such that $\alpha \leq u r^{2}<\beta$. where $\alpha$ and $\beta$ are numbers of order unity.

2. Approximate $\left(u r^{2}\right)^{-3 / 2}$.

3. Get the correct result by multiplying the approximation by $t=u^{3 / 2}$.

Because I want to keep my algorithm entirely in Fortran, I decided to use two tables for $u$ and $t$. The entries in $u$ are simply the power of 2 such that $1 \leq u r^{2}<2$. The entries in $t$ are $u^{3 / 2}$. The only problem is to figure out which table entries to use for a given input value.

The range reduction I use is based on the IEEE double precision number format [6]. Each number consists of 64 bits -1 sign bit, 11 exponent bits, and 52 fraction bits. In addition. there is an implicit 1 bit for normalized numbers.

If $I$ know the argument is positive. as it must be for the function I am interested in. I can extract the exponent by shifting the high order 32 bits of the floating-point number 20 bits to the right. In Fortran, this procedure requires that I EQLIVALENCE the double precision number to an integer or pass a double precision argument to a subroutine that uses it as an integer. Shifting the integer gives the index in the tables. Because there are only 11 bits to represent the exponent. I know my tables need only $2.0+8$ entries.

I could have coded my tables as DATA statements in the program. but I decided to ask the user to make a single call to set them up as is frequently done with Fourier transform routines. The code to build the tables is contained in the program in the Appendix.

Now that I have scaled the input to a modest range. I can do the approximation. The coefficients of the fit are easy to compute [5]. If I write the approximation of $\left(u r^{2}\right)^{-3 / 2}$ as

$$
f(x) \approx \frac{1}{2} c_{0}+\sum_{k=1}^{m} c_{k} T_{k}(x)
$$

where $x=2\left(u^{2}\right)-3$. the coefficients $c_{k}$ can be calculated from

$$
c_{k}=\frac{2}{\lambda} \sum_{j=1}^{l} f\left(x_{j}\right) T_{k}\left(x_{j}\right)
$$

where the $x_{j}$ are the zeros of $T_{V}(x), x_{j}=\cos [\pi j-$ $1 / 2 / \alpha]$. The change of variable from u $r^{2}$ to $x$ is needed because the Chebycher polynomials are orthogonal on the interval $[-1,1]$. If we choose $N \gg m$, the approximation $n$ ill be very close to the minimax polynomial.

It is important to use knowledge of the hardware in writing the code. I made my runs on an IBM RISC System 6000 Model $5+\dot{0}$. RISC machines nominally do all operations in one machine cycle, but in practice complicated operations are pipelined. On this machine, all floating-point additions and multiplications are treated as compound multiply/add operations [?]. An isolated operation takes two cycles. but a sequence of operations produces one result per cycle after a delay of two cycles. Thus, our goal is to produce compound operations that can be pipelined.

Figure 1 shows part of a function that the user invokes to do the Chebycher fit. The input value is 


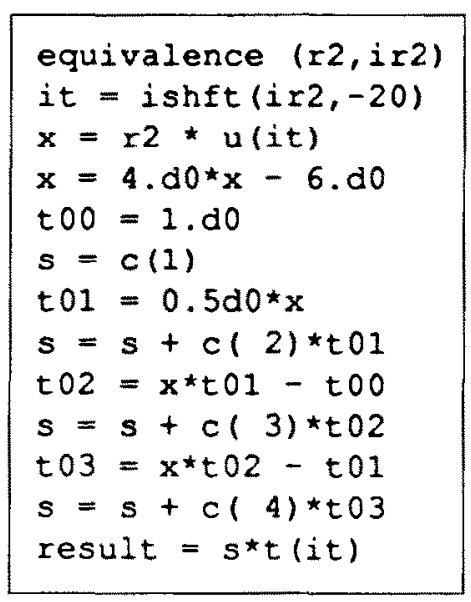

FICCRE 1 Colte to evaluate a four-term Chebrches fit. The input is r2. The tables $u$. $t$. and $c$ were calculated in the setup routine.

$\mathrm{r} 2$. The statement EQLTV ALENCE (r2, ir2) is needed because the shift function will only nork on an integer argument.* Only four terms are shown. but the extension to more is obvious.

After some set-up code to do the range reduction and shift the arguments into the range of the Chebycher polynomials, all operations but the last are multiply/adds. Lnfortunately, these operations are dependent on each other, so we are not making optimal use of the arithmetic pipeline. For example. the multiply/add that updates s cannot be started until the previous $t$ is ready. Also. computation of the next $t$ cannot be started until the previous one is done. However. we can overlap these two calculations so we expect each order of approximation to take an additional 3 cycles.

Table 2 summarizes the liming and accuracy results. The relative errors are measured using the direct calculation as the correct value. These errors are identical to the bounds computed by summing the absolute values of the dropped coefficients $[5]$.

The times are given in machine cycles per element. In each case I measured the elapsed time with a lock accurate to a few nanoseconds. The times reported are the smallest of 20 runs of 10,000 random inputs. Although there are some anomalies. most of the time it takes 3 cycles to add one more order to the approximation. The anomalies are caused by running out of registers and the performance of the memory when loading the coefficients.

One way to improve the overlap is to do more

* "Strung typing is nice. but it shouldn t be invincible:" X. L. Karp. private communication. 1985. than one evaluation on each pass through the loop, that is, unroll the loop. I experimentally determined that unrolling the loop eight ways gave me as much speed-up as I was going to get. The last column in Table 2 shows that adding one more term to the approximation costs less when the loop is unrolled about 2 cycles per term.

Is it worth using this approximation? It depends on the accuracy needed. The time stepping scheme will have some truncation error. Clearly, there is no point making the function evaluation more than an order of magnitude more accurate than this value.

Table 2 shows that single precision (about 8 digit) accuracy with around 10 terms can be obtained at a cost of 28 crcles, a third the cost of the direct computation. If more accuracy is needed. almost 16-digit accuracy can be obtained if 20 terms are used, but the speed-up over the direct calculation is small.

Anotler approach is to use Newton's method. It is based on finding the roots of some function, in this case

$$
f(y)=\frac{1}{y^{2}}-\left(r^{2}\right)^{3}
$$

The iteration is then

$$
y_{n+1}=y_{n}-\frac{f\left(y_{n}\right)}{f^{\prime}\left(y_{n}\right)}=\frac{1}{2} y_{n}\left(3-\left(r^{2}\right)^{3} y_{n}^{2}\right)
$$

where $n$ is the iteration index.

Newton's method is quadratically convergent when applied to a convex function such as the one in which we are interested [8]. This means that each iteration doubles the number of correct bits

Table 2. Summary of Measurements of Accuracy and Time for the Chebychev Approximation

\begin{tabular}{rccc}
\hline$m$ & Error & Rolled & Lnrolled $^{*}$ \\
\hline 0 & $7.2 \times 10^{-1}$ & 8 & 6 \\
2 & $3.1 \times 10^{-2}$ & 15 & 13 \\
4 & $1.1 \times 10^{-3}$ & 21 & 17 \\
6 & $3.8 \times 10^{-1}$ & 28 & 21 \\
8 & $1.3 \times 10^{-6}$ & 33 & 25 \\
10 & $4.2 \times 10^{-8}$ & 37 & 28 \\
12 & $1.3 \times 10^{-4}$ & 42 & 33 \\
14 & $4.2 \times 10^{-11}$ & 59 & 38 \\
16 & $1.3 \times 10^{-12}$ & 60 & 43 \\
18 & $4.1 \times 10^{-14}$ & 50 & 48 \\
20 & $3.2 \times 10^{-15}$ & 53 & 50 \\
\hline
\end{tabular}

* Lnrolled refers to loops unrolled eight ways. Times are in machine cycles per element. 


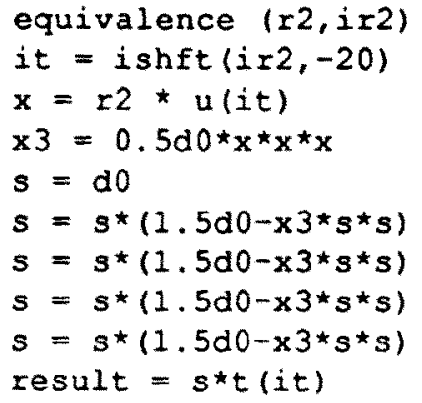

FIGURE 2 Code for four lewton iterations. The arrays $u$ and $t$ were calculated in the setup routine.

in the result. We only need to get a reasonably accurate first guess. If we use the same range reduction as before, a reasonable first guess would be the function evaluated near the midpoint of the range. In fact, I chose to use a zero th order Chebychev fit for the first guess.

Figure 2 shows the key part of the function invoked by the user. We see that this code will not use the hardware as effectively as the Chebycher code. Each Newton iteration has a multiplication. a multiply/add. and a final multiplication for a total of 6 crcles.

Table 3 shows the convergence and time for rolled and unrolled loops. If the hop is not unrolled. Newton's method takes 6 cycles per iteration as predicted: it takes only about 3 if the loop is unrolled. Single precision accuracy is achieved in about 29 cycles per element and double precision in $3+$ cycles per element.

Is it worth using Nenton's method? Yes it is unless you need the last fen hits correct. Nithout doing arithmetic in a higher precision the loss of a few bits of accuracs is inevitable. However. the simplicity of the code and its speed are in it favor.

Table 3. Summary of Measurements of Accuracy and Time for the Newton Method

\begin{tabular}{cccc}
\hline$n$ & Error & Rolled & Lnrolled* \\
\hline 1 & $5.2 \times 10^{-1}$ & 17 & $1+$ \\
2 & $3.3 \times 10^{-1}$ & 23 & 16 \\
3 & $1.4 \times 10^{-1}$ & 30 & 20 \\
4 & $3.0 \times 10^{-2}$ & 36 & $2+$ \\
5 & $1.3 \times 10^{-3}$ & +2 & 26 \\
6 & $2.7 \times 10^{-6}$ & 48 & 29 \\
7 & $1.1 \times 10^{-11}$ & $5+$ & 32 \\
8 & $+3 \times 10^{-16}$ & 60 & $3+$ \\
\hline
\end{tabular}

* Cnrolled refers to hops unrolled eight wavis. Times are in machine cydes per eltutent.
We can do considerably better by making two changes to the Newton's method code. First of all. note the small improvement in the first fex iterations. A better first guess nould reduce the number of Newton iterations dramatically. I chose a six-term Chebycher fit that results in single precision accuracy with one Newton iteration and double precision with two.

There is another trick that can be used if only six terms are to be used in the polynomial approximation-compute the coefficients of the powers of $x$. This approach is not recommended in general because of the potential round-off errors when the coefficients are combined. Here there is no need to worr because the Newton iteration will tolerate such errors. The monomial coefficients are

$$
\begin{aligned}
& d_{0}=\frac{1}{2} c_{1}-c_{2}+c_{4} \\
& d_{1}=c_{1}-3 c_{3}+5 c_{3} \\
& d_{2}=2 c_{2}-8 c_{4} \\
& d_{3}=4 c_{3}-20 c_{3} \\
& d_{4}=8 c_{4} \\
& d_{3}=16 c_{3}
\end{aligned}
$$

If we use Horner's rule to evaluate the approximation.

$$
s=d_{0}+x\left(d_{1}+x\left(d_{2}+x\left(d_{3}+x\left(d_{4}+x d_{3}\right)\right)\right)\right.
$$

the polynomial evaluation is all multiply/adds. The key part of the code is shom in Figure 3.

Table + summarizes the performance results. The see that ne get single precision ancuracy in only 19 cycles and double precision in 23 escles. Because this algorithm outperformi the diret evaluation by a factor of nearly + . an X-hody coole

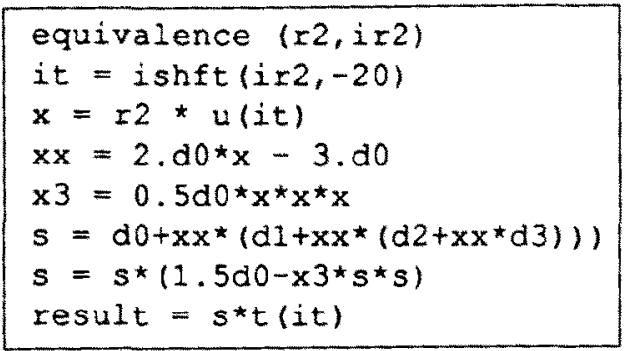

FIGURE 3 Code for the hybrid method. A third orter Chebychev fit and one Vewton iteration are slown. The coefficients $d$ and the arrars $u$ and $t$ were calculated in the setup routine. 
Table 4. Summary of Measurements of Accuracy and Time for the Hybrid Method*

\begin{tabular}{rccc}
\hline$n$ & Error & Rolled & Lnrolled \\
\hline 0 & $7.2 \times 10^{-1}$ & 9 & 7 \\
1 & $1.6 \times 10^{-1}$ & 10 & 10 \\
2 & $3.1 \times 10^{-2}$ & 12 & 11 \\
3 & $6.0 \times 10^{-3}$ & 13 & 12 \\
4 & $1.1 \times 10^{-3}$ & 16 & 13 \\
5 & $2.1 \times 10^{-4}$ & 17 & 14 \\
11 & $6.6 \times 10^{-13}$ & 23 & 19 \\
12 & $6.6 \times 10^{-15}$ & 30 & 23 \\
\hline
\end{tabular}

* The first wix row are the order of the Chebrcher fit: the last two are the Veaton iterations. Linrolled refers to loups unrolled eighn ways. Times are in machine cycles per element.

using this approach should run considerably faster.

The referee pointed out one more trick that reduces the times in Table + by one cycle per element. The Chebychev polynomials are evaluated on the interval $-1 \leq x<1$ while we have done a range reduction to $1 \leq u r^{2}<2$. The variable $\mathrm{xx}$ is used to do the required change of variables. Examination of the code shows that $\mathrm{xx}$ can be substituted into the polynomial approximation for $\mathbf{s}$. the terms can be arranged, and the new coefficients precomputed. These new coefficients, call them $g_{k}$ are related to the $d_{k}$ by

$$
\begin{array}{lrr}
g_{0}=d_{0}-3 d_{1}+9 d_{2}-27 d_{3}+81 d_{4}-2+3 d_{5} \\
g_{1}= & 2 d_{1}-12 d_{2}+54 d_{3}-216 d_{4}+810 d_{3} \\
g_{2}= & +d_{2}-36 d_{3}+216 d_{4}-1080 d_{5} \\
g_{3}= & 8 d_{3}-96 d_{4}+720 d_{3} \\
g_{4}= & 16 d_{4}-2+0 d_{5} \\
g_{5}= & 32 d_{3}
\end{array}
$$

Table 5. Coelficients of the Minimax Fit*

\begin{tabular}{rrrr}
\hline$k$ & $c$ & \multicolumn{1}{c}{$l$} & \multicolumn{1}{c}{$g$} \\
\hline 0 & 0.60800336 & $0.5+4+17+1$ & $7.05+52+70$ \\
1 & -0.30951280 & $-0.2722756+$ & $-1+.85088557$ \\
2 & 0.06613347 & 0.11188678 & $1+.70832310$ \\
3 & -0.01321311 & $-0.0+32+377$ & -7.83703555 \\
4 & $0.0025+752$ & 0.02038017 & $2.1709+576$ \\
5 & $-0.000+80+3$ & -0.00768693 & $-0.2+59817+$ \\
\hline
\end{tabular}

* Three forms are given: $c$ are the coefficients of the orthogonal polyomials: a are the corfficitnts of the monomials on $[-1,1]$ : gre are coeffirients of the monomials on [1,2.
We now evaluate the polynomial

$$
\begin{aligned}
s= & g_{0}+\left(u r^{2}\right)\left(g_{1}\right. \\
& \left.+\left(u r^{2}\right)\left(g_{2}+\left(u r^{2}\right)\left(g_{3}+\left(u r^{2}\right)\left(g_{4}+\left(u r^{2}\right) g_{5}\right)\right)\right)\right) .
\end{aligned}
$$

The complete subroutine, including the set-up code, is shown in the Appendix; the coefficients are shown in Table 5 .

\section{CONCLUSIONS}

How can I beat the performance of a highly tuned sustem routine with Fortran code? Simple-I cheat.

I cheat in a number of wavs. First of all. I evaluate the function directly rather than in pieces. Second. I cheat by not getting the last few bits right. Finally, I cheat by not doing any error checking. ( 1 could take the absolute value of the input at the cost of one additional cycle.) However, the output value is accurate for any floatingpoint input. Very large input values produce denormalized results: very small input values produce floating point infinity as they should.

If your machine supports the IEEE double extended format [6], a format with at least 64 bits in the fraction that is usually reserved for data kept in the registers, you can get the last few bits right using a simple trick. Compute the array $t$ in extended precision. but store it as two double precision numbers, $t(1, i)$ and $t(2, i)$. Then the final scaling becomes $s^{*}(t(1, i)+t(2, i))$. I could not check the accuracy because the RISC Srstem $/ 6000$ does not support the double extended format, but the change added only 1 cycle per result to the time of the unrolled loop.

Is it worth the effort and worry to use this new approach? If your calculation is ypical and spends $3 / 4$ of its time evaluating the acceleration, speeding up this one line of code by a factor of 3 will cut your total run time in hall.

\section{ACKNOWLEDGMENTS}

I would like to thank Vivek Sarkar for helping me understand the RS/6000 instruction scheduling, Rad Olson and Bill Swope for trying to comince me I could not beat the system functions (I love a challenge), and the referee for several good ideas. 


\section{APPENDIX 1}

\section{Sample Code}

This Appendix contains the complete version of the code as measured. For the sake of space, I changed the loop unrolling from eight-way to twoway. Some points are worthy of note.

If working with single precision data, change the shift to ishft $(x, 23)$ because IEEE single precision only uses 8 bits for the characteristic.
Also, the center for the arrays $t$ and $u$ should be at 127.

Some srstems do not allow continuation of execution following an overflow. Because $r^{-3 / 2}$ will underflow and overflow at the boundaries of the floating-point arithmetic, adjust loop limita on these machines. However, make sure that the table is filled properly. Lnderlow should produce a true zero and overflow should produce foating point infinity.

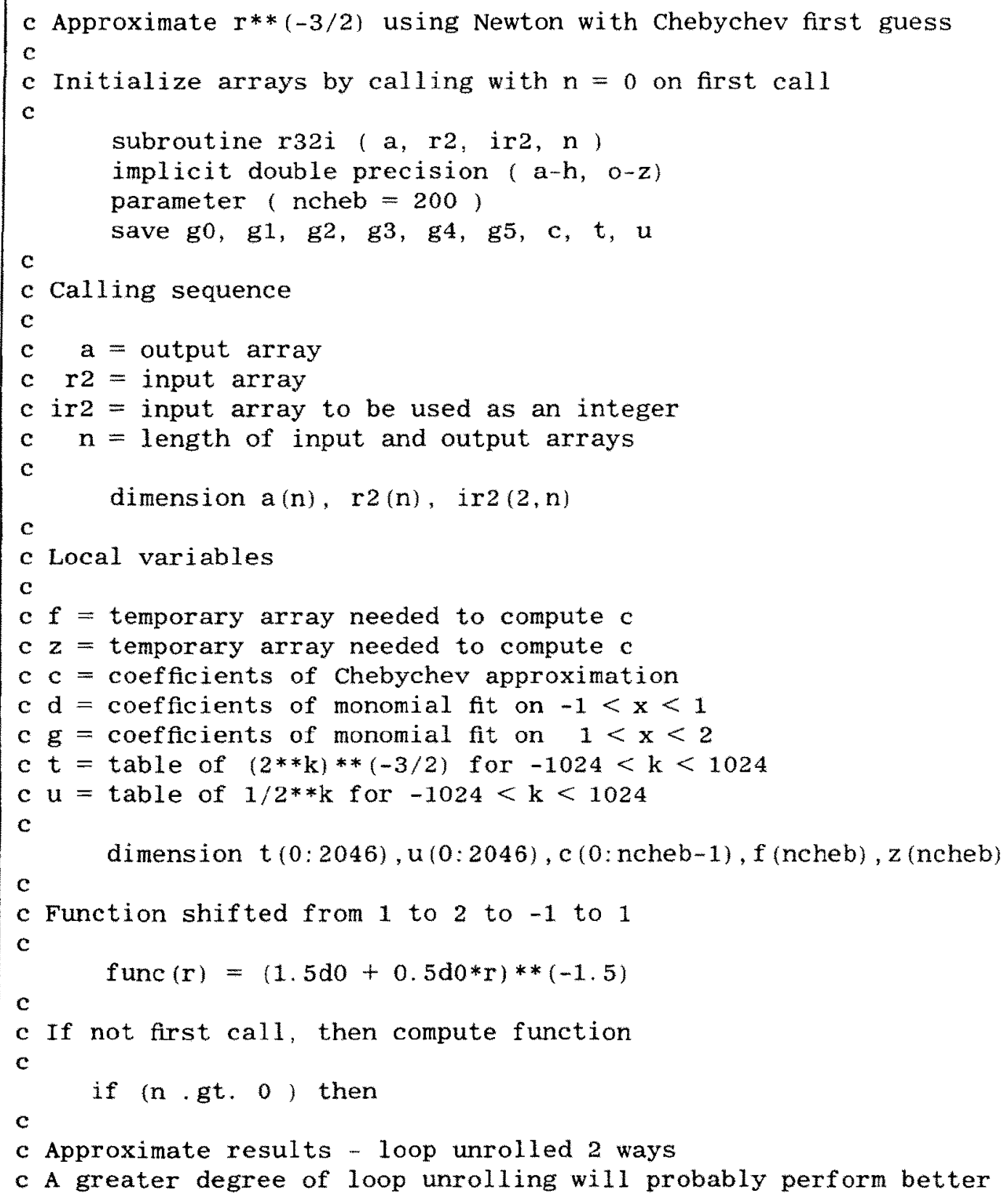




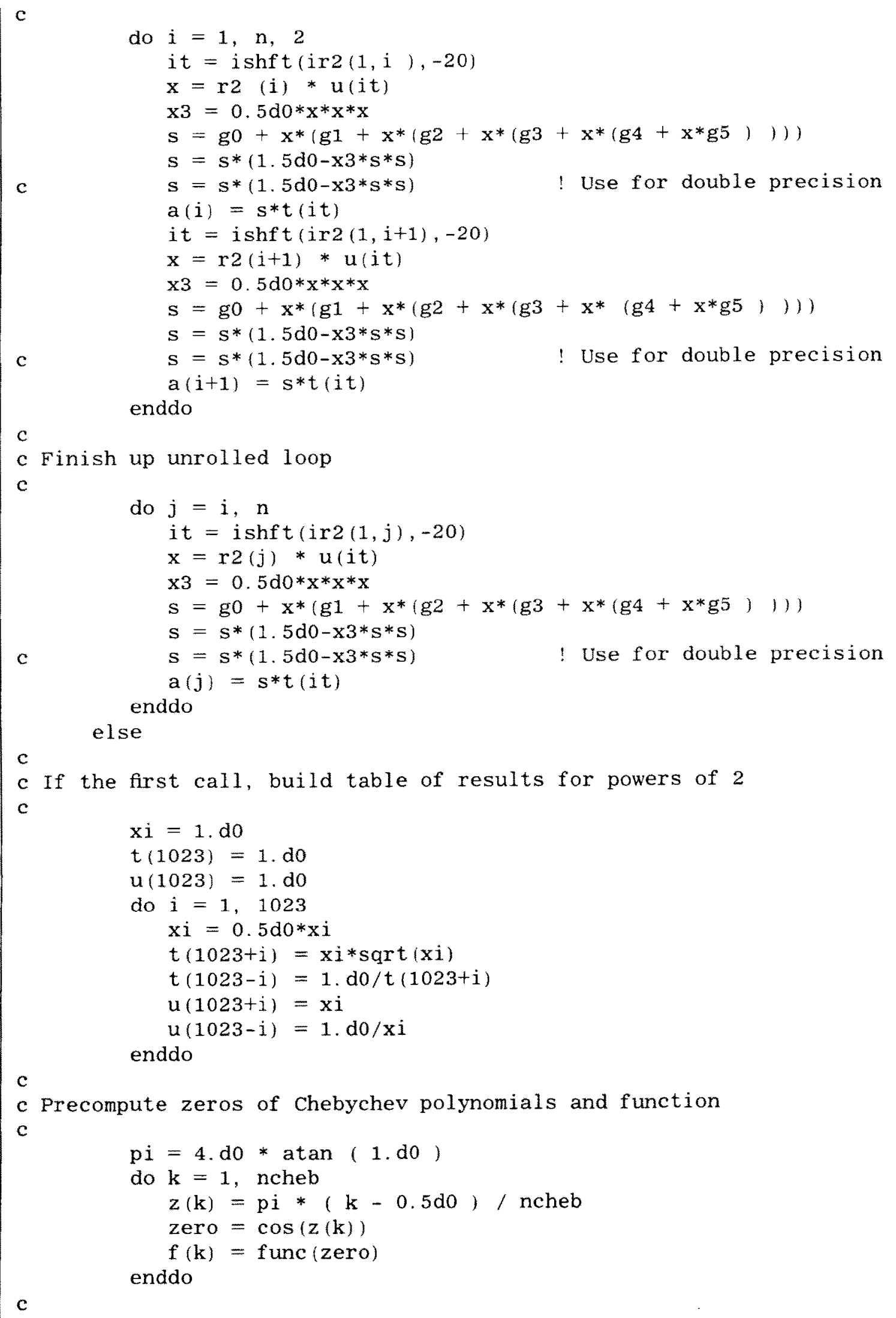




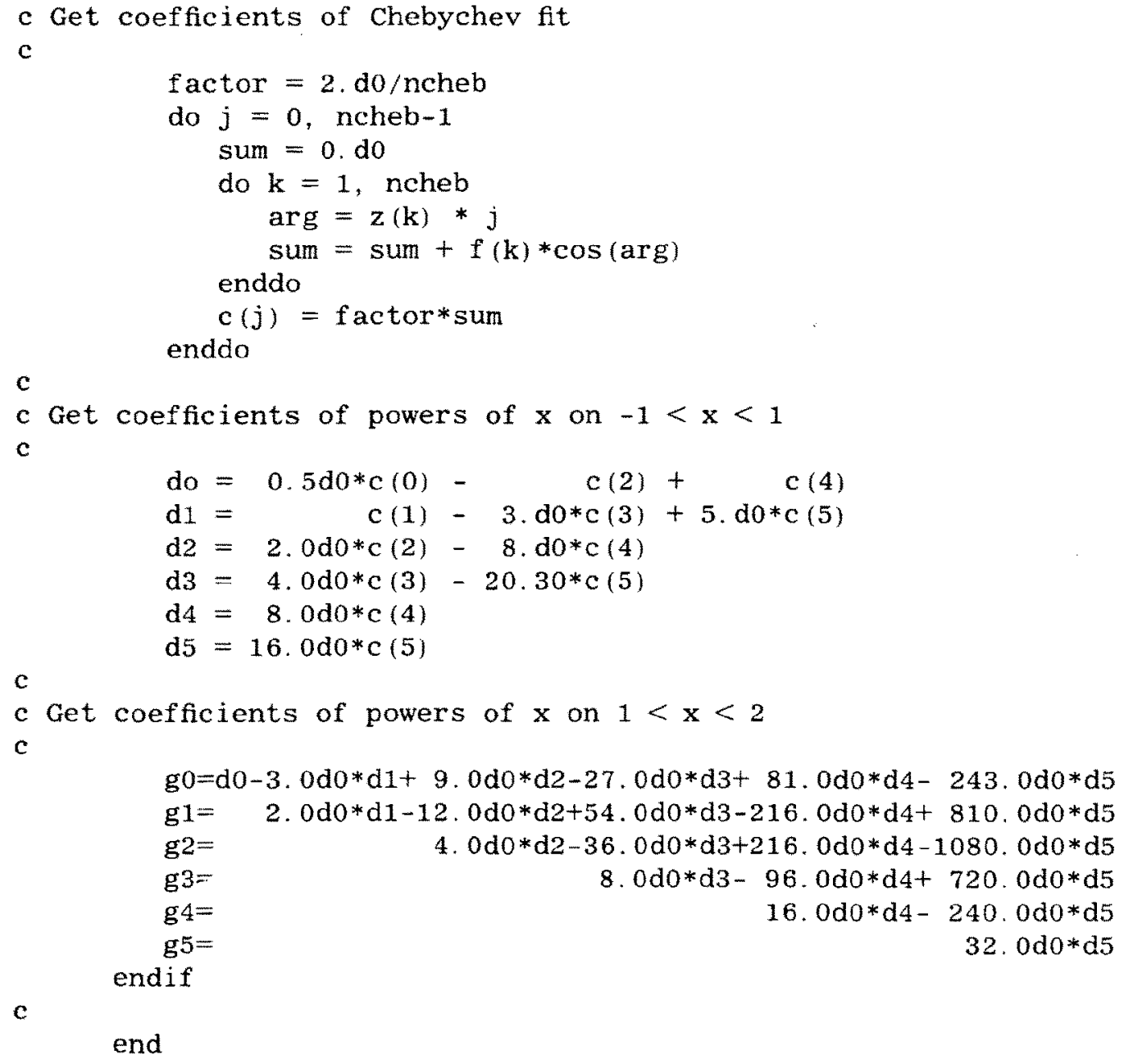

\section{REFERENCES}

[1] R. W. Hockney and J. W. Eastwood. Computer Simulation Lising Particles. New York: MeGrawHill. 1981.

[2] J. A. Sellwod, Annual Revieus of Astronomy and Astrophysics, vol. 25. Palo Alto. CA: Annual Re:views. Inc., 198? pp. 151-186.

[3] J. Barnes and $P$. Hut $\cdots$ A hierarchical O Nogr force-calculation algorithm." Nature, vol. 32+. pp. $4+6-4+9.1986$.

[+] M. P. Allen and D. J. Tildesler. Computer Simulation of Liquids. Oxford. L $\mathrm{K}$ : Oxford Cniversity Press, 1987.
[5] II. H. Press. B. P. Flannery. S. A. Teukolikr. and W. T. Vetterling. Vumerical Rectpes. New lork: Cambridge Lniversity Press. 1986. pp. 1+7-151.

[6] American National Standards Institute. Inc. IEEE Standard for Binary Floating-Point Arithmetic. Technical Report ANSl/LEE Std 75t-1985. IlEE. 3+5 East +?th Sreet. New lork. NY 1001?. 1985.

[7] B. Olsson. R. Montoye. P. Markstein. and Y. Siryen Pluu, RISC System/6000) Flocting-point Lnit. IBH NY: RISC: System/6000 Technologr. 1990, pp. $3+-+2$.

[8] J. M. Ortega Numerical Analsis. Yew York: Academic Press. 1972. pp. 155-158. 

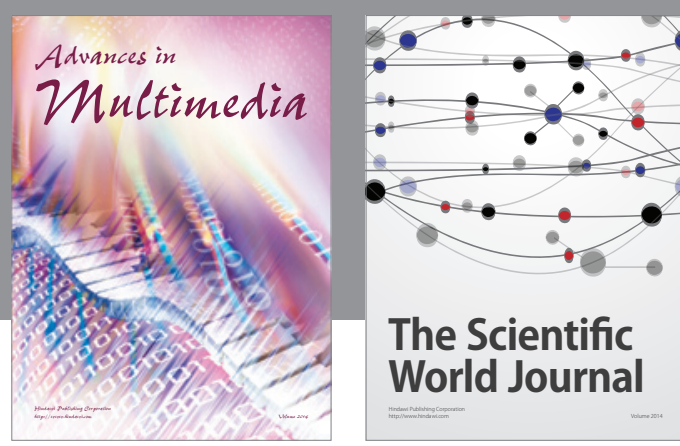

The Scientific World Journal
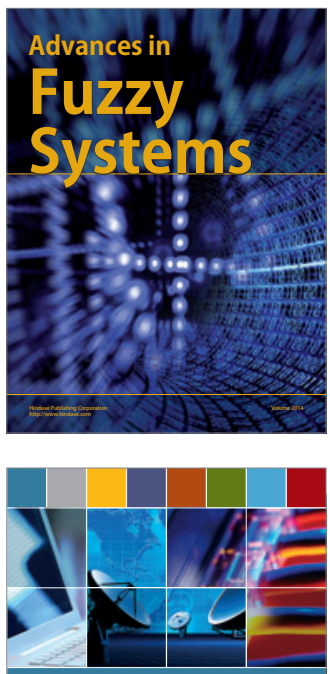

Computer Networks and Communications
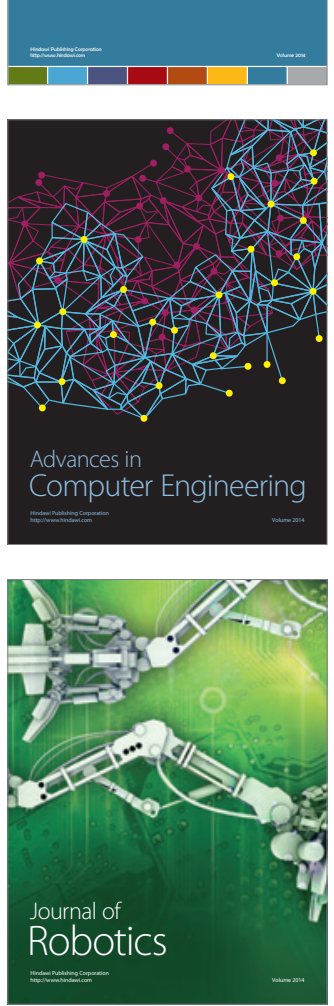
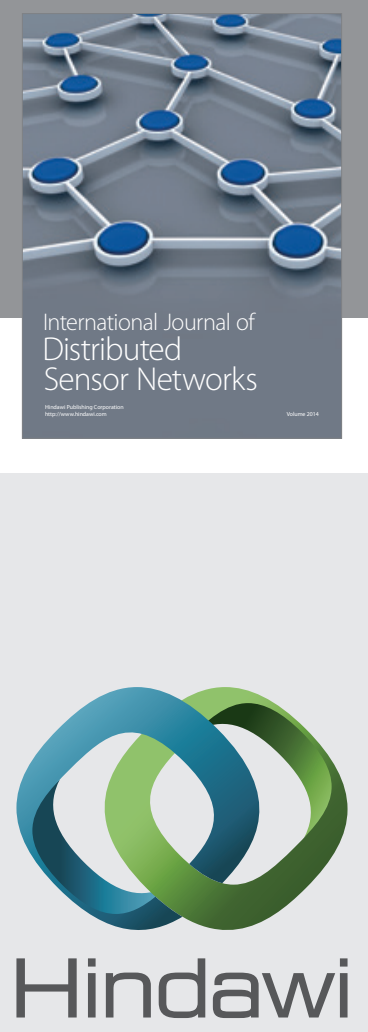

Submit your manuscripts at

http://www.hindawi.com
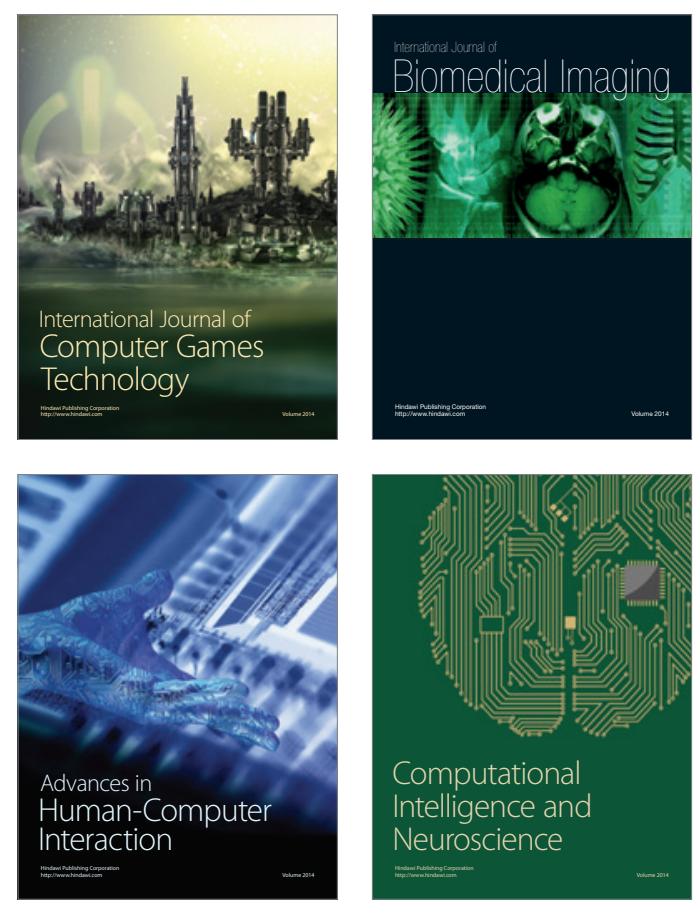
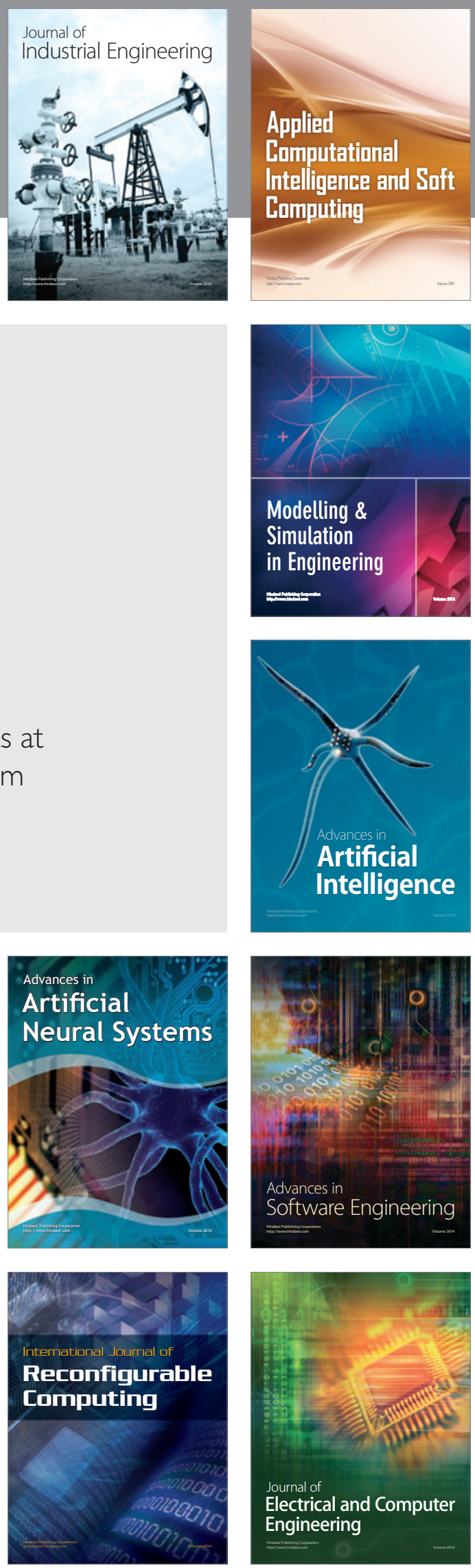